\title{
Slow Invariant Manifold of Brusselator Model
}

\author{
A. K. M. Nazimuddin ${ }^{\mathrm{a}, *}$, Md. Showkat Ali ${ }^{\mathrm{b}}$ \\ ${ }^{a}$ Department of Mathematical and Physical Sciences, East West University, Dhaka-1212, \\ Bangladesh. \\ ${ }^{\mathrm{b}}$ Department of Applied Mathematics, University of Dhaka, Dhaka-1000, Bangladesh.
}

Received: 30 August 2019; Accepted: 15 November 2019; Published: 08 April 2020

\begin{abstract}
The slow invariant manifold is a unique trajectory of the dynamical system that describes the long-time dynamics of the system's evolution efficiently. Determining such manifolds is of obvious importance. On one hand they provide a basic insight into the dynamics of the system, on the other hand they allow a reduction of dimension of the system occurs on the invariant manifold only. If the dimension of the invariant manifold is sufficiently low, this reduction may result in substantial savings in computational costs. In this paper, differential geometry based new developed approach called the flow curvature method is considered to analyse the Brusselator model. According to this method, the trajectory curve or flow of any dynamical system of dimension considers as a curve in Euclidean space of dimension. Then the flow curvature or the curvature of the trajectory curve may be computed analytically. The set of points where the flow curvature is null or empty defines the flow curvature manifold. This manifold connected with the dynamical system of any dimension directly describes the analytical equation of the slow invariant manifold incorporated with the same dynamical system. In this article, we apply the flow curvature method for the first time on the two-dimensional Brusselator model to compute the analytical equation of the slow invariant manifold where we use the Darboux theorem to prove the invariance property of the slow manifold.
\end{abstract}

Index Terms: Dynamical System, Differential Geometry, Flow Curvature Method, Slow Manifold.

(C) 2020 Published by MECS Publisher. Selection and/or peer review under responsibility of the Research Association of Modern Education and Computer Science

* Corresponding author. Tel.: +8801920179472

E-mail address: nazimuddin@ewubd.edu 


\section{Introduction}

Singularly perturbed systems can have invariant manifolds where the trajectories of the flow move slowly and these slow manifolds are invariant with respect to the flow [1, 2, 3]. Several methods have been developed to find out the analytical slow manifold equations of the singularly perturbed systems. In $[4,5,6,7,8,9,10$, 11], introduces the geometric singular perturbation technique to establish the existence of the slow manifold equation along with the local invariance of the slow manifold for the singularly perturbed system. In the case of non-singularly perturbed system like that of Brusselator model this technique fails to provide the slow manifold.

The flow curvature method $[12,13,14,15,16]$ is the new method in recent publications for computing the analytical implicit equation of the slow manifold. This method can be applied to any autonomous or nonautonomous dynamical systems in $\mathrm{n}$ dimensions whether it is singularly perturbed or not. Recent applications of the flow curvature method of the singularly perturbed systems are FitzHugh-Nagumo model, Van der pol model, Chua's model, etc and the applications of the flow curvature method of the non-singularly perturbed systems are Lorenz model, Rikitake model, etc. [17] used the flow curvature method to construct the slow invariant manifold of the heartbeat model. In [18], author developed the slow invariant manifold analytical implicit equation of the generalized Lorenz-Krishnamurthy model and conservative generalized LorenzKrishnamurthy model. The most important feature of this method is that, the flow curvature manifold directly gives us the analytical equation of the slow manifold. Without using any asymptotic expansions, this method allows us to find the flow curvature manifold and hence slow invariant manifold equation. To the best of our knowledge, this method is the best to find the analytical equation of the slow invariant manifold for any dimensional dynamical system.

We consider an activator-inhibitor Brusselator model which represents an autocatalytic oscillating chemical reaction. In paper [19], authors discussed the asymptotic behaviour of the solutions of the Brusselator model numerically. In paper [20], author studied various types of pattern formation of the Brusselator model arising in chemical reactions with the numerical investigation. In paper [21, 22], authors investigated periodic traveling wave solutions of the Brusselator model using numerical bifurcation analysis. We study the slow invariant manifold of the Brusselator model for the first time and this study advances the field from the previous related work. So, our goal of the present paper is to analyze the local behaviour of the slow-fast dynamical Brusselator model by using the analytical implicit equation of the slow invariant manifold where we use Darboux theory to prove the invariance property of the slow manifold of the Brusselator model. To simulate the Brusselator model, we use MATHEMATICA as a software tool.

The remainder part of this paper is organized as follows. In section 2, we describe the nonlinear slow-fast Brusselator model. We discuss the flow curvature method based on the differential geometry in section 3 . We also present the Darboux theorem in this section which represents the invariance condition of the slow manifold of a dynamical system. In section 4, we express the slow manifold analytical equation of the Brusselator model. Some conclusions are given in section 5.

\section{Brusselator Model}

The Brusselator system describes the following chemical reactions [23].

$$
\begin{aligned}
& A \rightarrow U, B+U \rightarrow V+D, \\
& 2 D+V \rightarrow 3 U, U \rightarrow E
\end{aligned}
$$

Since it is important to consider at least a cubic nonlinearity in the rate equations, so the non-dimensional form of the Brusselator model (temporal only) is as follows: 


$$
\begin{aligned}
& \frac{d x}{d t}=a-(b+1) x+x^{2} y, \\
& \frac{d y}{d t}=b x-x^{2} y,
\end{aligned}
$$

where $u$ and $v$ are the dimensionless concentration called activator and inhibitor and $a$ and $b$ are the kinetic parameters. The equilibrium point for the system (1) is $(a, b / a)$.

\section{Flow Curvature Method}

In this part, we briefly discuss the flow curvature method in terms of differential geometry. This method uses the properties of curvatures of trajectory curve or flow of the dynamical system. Using this method, one can define the flow curvature manifold corresponding to the dynamical system. Any $n$-dimensional dynamical system can have the $(n-1)$ dimensional flow curvature manifold that means flow curvature manifold contains the information about the flow with highest curvature.

\subsection{Analytical Implicit Equation of the Slow Manifold of the Dynamical System}

Invariant manifold implies a very significant role to explain the stability as well as dynamical behavior of a system, especially for a slow-fast dynamical system. Although geometric perturbation technique is well known to find the analytical equation of slow manifold, the main difference between geometric perturbation technique and the flow curvature method is that it neither uses asymptotic expansions nor eigenvectors. Another difference is that this method can be used for any dynamical system which may or may not singularly perturbed.

Proposition 3.1 The set of points where the curvature of the flow of the model (1) vanishes represented by the following flow curvature manifold equation of the dynamical system.

$$
\psi(\vec{X})=\operatorname{det}(\overrightarrow{\dot{X}}, \vec{X})=0
$$

Proof See $[13,14]$

Note that for any n-dimensional dynamical system, maximum $(n-1)^{\text {th }}$ flow curvature is possible.

Proposition 3.2 The flow curvature manifold of the dynamical system (1) directly provides its implicit analytical equation of the slow manifold.

Proof See $[13,14]$

\subsection{Darboux Invariance Theorem}

According to [24, 25], the concept of the invariant manifold is first introduced by G. Darboux (1878, p. 71). We consider the trajectories of the dynamical system (1) is represented by a motion of a point in a two dimensional space and the coordinates of the point is $\vec{X}=(x, y)$ and the velocity vector of this point is $\vec{V}=(\dot{x}, \dot{y})$.

Proposition 3.3 Consider $\psi(\vec{X})=\operatorname{det}(\vec{X}, \vec{X})=0$ is a slow manifold of the dynamical system (1) where $\psi$ is a first time continuously differentiable function, then this manifold is invariant with respect to the flow of (1) if 
there exist a first time continuously differentiable function called cofactor $C(\vec{X})$ which satisfies the following equation:

$$
\mathrm{L}_{\vec{V}} \psi(\vec{X})=C(\vec{X}) \psi(\vec{X})
$$

with the Lie derivative defined as the following:

$$
\mathrm{L}_{\vec{V}} \psi(\vec{X})=\vec{V} \cdot \vec{\nabla} \psi=d \psi / d t
$$

\section{Proof See $[13,14]$}

\section{The Slow Invariant Manifold of the Brusselator model}

According to the flow curvature method, the trajectory curves of any dynamical system which may or may not singularly perturbed considered the curves in the Euclidean space. We consider the system model (1) as the slow-fast dynamical system.

We use the parameter values of (1) as mentioned in Table 1 and for the numerical simulation, we consider the range of the state variables connected with the dynamical system (1) as the following

$$
\begin{aligned}
& {\left[\mathrm{x}_{\min }, \mathrm{x}_{\max }\right]=[-.1,10] ;} \\
& {\left[\mathrm{y}_{\min }, \mathrm{y}_{\text {max }}\right]=[0,20] ;}
\end{aligned}
$$

Table 1. Typical parameter values of (1) for the numerical computations.

\begin{tabular}{lcc}
\hline Parameters & $a$ & $b$ \\
\hline Values & 3.0 & 14.0 \\
\hline
\end{tabular}

By putting right hand side parts of the dynamical system (1), that is,

$$
\begin{aligned}
& a-(b+1) x+x^{2} y=0, \\
& b x-x^{2} y=0,
\end{aligned}
$$

we obtain two following graphs for the two null-clines of the system (1).

Thus, we get the following fixed point by solving the system (2)

$$
\mathrm{x}_{1}=3 ; \mathrm{y}_{1}=\frac{14}{3} .
$$

We use explicit Runge-Kutta method [26] to solve the model (1) numerically where we use $\left(x_{0}, y_{0}\right)=(1,1)$ as an initial point. Fig.2 shows the phase diagram represented by (1) where $t$ ranges from 80 to 100 . Also, the purple point in the fig.2 indicate the fixed point of the model (1). 


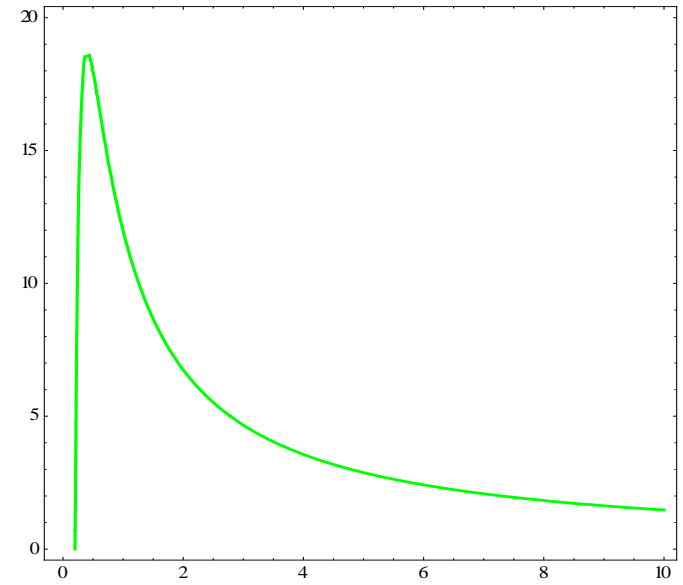

(a)

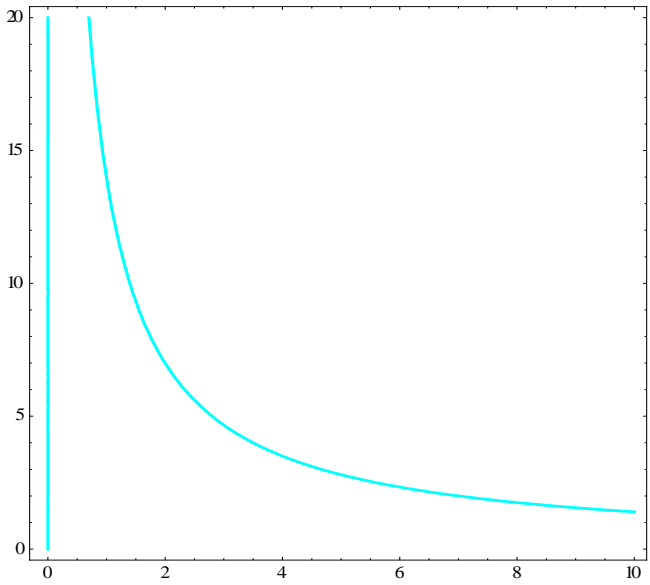

(b)

Fig.1. Nullclines of the model (1). (a) first equation of the system (2), (b) second equation of the system (2).

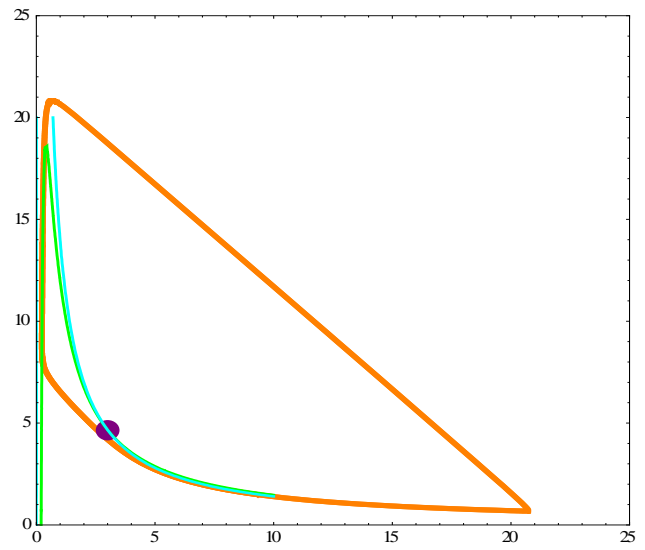

Fig.2. Phase plot analysis for the model (1) along with the two nullclines and single fixed point obtained from the same model.

Now, in order to calculate the flow curvature manifold of the model (1) using the flow curvature method, we need the velocity and acceleration because of our 2-dimensional dynamical model. The velocity vector field of the model (1) can be represented by the following way.

$$
\overrightarrow{\mathrm{V}}=\left\{3-15 x+x^{2} y, 14 x-x^{2} y\right\}
$$

The Jacobian matrix corresponding to the model (1) may be written as

$$
J=\left(\begin{array}{cc}
15+2 x y & x^{2} \\
14-2 x y & -x^{2}
\end{array}\right)
$$


Now we get the acceleration vector by using the formula $\vec{A}=J \vec{V}$ and hence, we obtain

$$
\vec{A}=\left\{x^{2}\left(14 x-x^{2} y\right)+(-15+2 x y)\left(3-15 x+x^{2} y\right),-x^{2}\left(14 x-x^{2} y\right)+(14-2 x y)\left(3-15 x+x^{2} y\right)\right\}
$$

So, the analytical implicit equation of the slow manifold of the model (1) can be written as

$$
\psi(x, y, z)=126-630 x-42 x^{3}+14 x^{4}-18 x y+135 x^{2} y-15 x^{3} y+3 x^{4} y-x^{5} y-6 x^{3} y^{2}+x^{4} y^{2}=0
$$

In Fig.3(a) shows the graphical representation of the analytical implicit equation of the slow manifold represented by the equation (3) and Fig.3(b) represents the slow manifold as well as phase space diagram in the same graph.

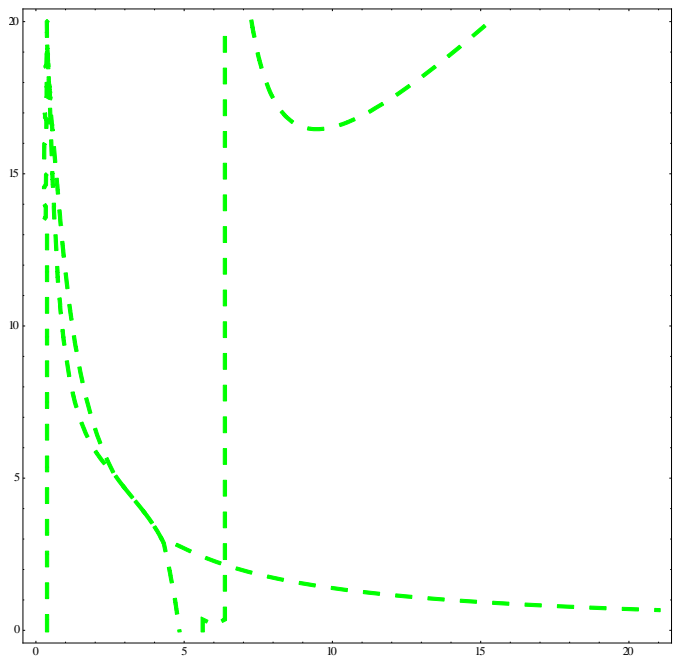

(a)

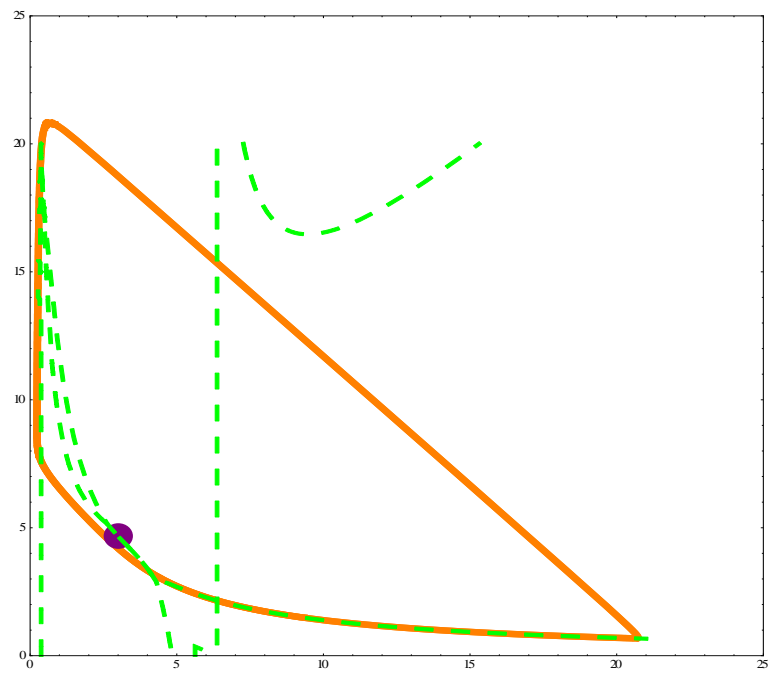

(b)

Fig.3. (a) Graphical representation of the slow manifold analytical equation of the model (1) using flow curvature method, (b) Graphical representation of the slow manifold analytical equation along with the phase diagram represented by (1).

The Lie derivative of the slow manifold function is then evaluated as the following by using the Darboux invariance theory to establish the flow curvature invariance of the equation (3). We first find the normal vector of the flow curvature manifold and we get

$$
\vec{\nabla} \psi=\left\{\begin{array}{l}
-630-126 x^{2}+56 x^{3}-18 y+270 x y-45 x^{2} y+12 x^{3} y-5 x^{4} y-18 x^{2} y^{2}+4 x^{3} y^{2}, \\
-18 x+135 x^{2}-15 x^{3}+3 x^{4}-x^{5}-12 x^{3} y+2 x^{4} y
\end{array}\right\}
$$

Now according to proposition 3.3, we compute Lie derivative of the slow manifold as follows

$$
\begin{aligned}
& \mathrm{L}_{\overrightarrow{\mathrm{v}}} \psi=-1890+9450 x-630 x^{2}+3948 x^{3}-1050 x^{4}+42 x^{5}-14 x^{6}-54 y+ \\
& 1080 x y-4815 x^{2} y+729 x^{3} y-624 x^{4} y+174 x^{5} y-3 x^{6} y+x^{7} y-72 x^{2} y^{2}+ \\
& 552 x^{3} y^{2}-105 x^{4} y^{2}+24 x^{5} y^{2}-7 x^{6} y^{2}-18 x^{4} y^{3}+4 x^{5} y^{3}
\end{aligned}
$$


In Fig.4(a) shows the graphical representation of the equation $\mathrm{L}_{\vec{v}} \psi=0$ that means the graphical representation of the flow curvature invariance manifold where the rate of change of $\psi(x, y, z)$ is equal to zero and Fig.4(b) shows the combined graph of the invariance manifold and phase space plot.

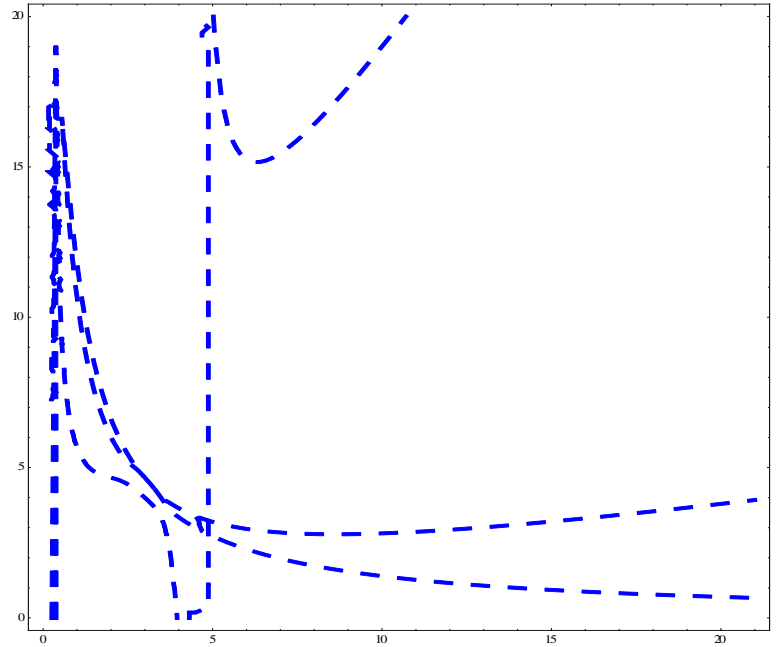

(a)

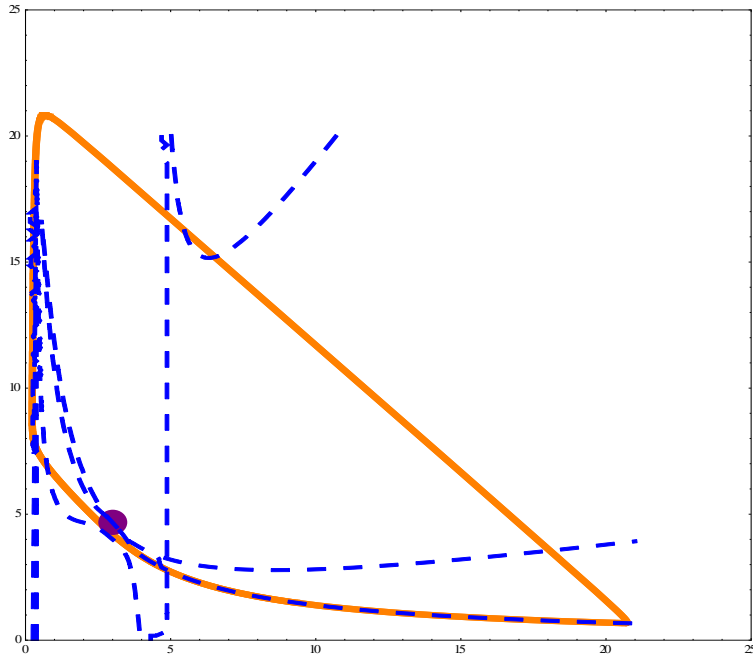

(b)

Fig.4. (a) Graphical representation of the invariance equation of the slow manifold analytical equation of the model (1) according to the Darboux theorem (b) Graphical representation of the invariance equation of the slow manifold analytical equation along with the phase diagram represented by (1).

\section{Conclusion}

Brusselator model exhibits a slow-fast structure where a solution from some arbitrary initial condition rapidly relaxes toward some attracting invariant manifold where the dynamics progresses slowly. In this article, we applied a method called flow curvature method to the Brusselator model which reflects the applications of differential geometry in the dynamical system. Slow invariant manifolds of the Brusselator model are calculated to obtain a model reduction. Since this manifold comprises the time derivatives of the velocity vector field and hence it receives knowledge about the dynamics of the corresponding system. We used flow curvature method to determine the analytical equation of the slow invariant manifold of the model. We performed a numerical simulation of the Brusselator model by using explicit Runge-Kutta method. We also used Darboux invariance theorem to show the invariance of the slow manifold.

\section{Acknowledgements}

The author wishes to thank Dr. Md. Showkat Ali for his guidance and supervision during the research period. 


\section{References}

[1] Andronov, A.A., Chaikin, S.E (1937). Plane Theory of Oscillators, I, Moscow.

[2] Tikhonov, A.N. (1948). On the dependence of solutions of differential equations on a small parameter, Mat. Sbornik N. S., 31:575-586.

[3] Levinson, N., (1949). A second-order differential equation with singular solutions, Ann. Math, 50:127153.

[4] Fenichel, N. (1971). Persistence and smoothness of invariant manifolds for flows, Indiana Univ. Math. J, 21:193-225.

[5] Fenichel, N. (1974). Asymptotic stability with rate conditions, Indiana Univ. Math. J, 23:1109-1137.

[6] Fenichel, N. (1977). Asymptotic stability with rate conditions II, Indiana Univ. Math. J, 26:81-93.

[7] Fenichel, N. (1979). Geometric singular perturbation theory for ordinary differential equations, J. Differ. Equ., 31: 53-98.

[8] Wasow, W.R. (1965). Asymptotic Expansions for Ordinary Differential Equations, Wiley-Interscience, New York.

[9] Cole, J.D. (1968). Perturbation Methods in Applied Mathematics, Blaisdell, Waltham.

[10] O’Malley, R.E. (1974). Introduction to Singular Perturbations, Academic Press, New York.

[11] O’Malley, R.E. (1991). Singular Perturbations Methods for Ordinary Differential Equations, Springer, New York.

[12] Ginoux, J.M. and Rossetto, B. (2006). Differential geometry and mechanics applications to chaotic dynamical systems, Int. J. Bifurc. Chaos, 4(16): 887-910.

[13] Ginoux, J.M., Rossetto, B. and Chua, L.O. (2008). Slow invariant manifolds as curvature of the flow of dynamical systems, Int. J. Bifurc. Chaos, 11(18): 3409-3430.

[14] Ginoux, J.M. (2009). Differential geometry applied to dynamical systems, In: World Scientific Series on Nonlinear Science, Series A, 66, World Scientific, Singapore.

[15] Ginoux, J.M.and Llibre, J. (2011). The flow curvature method applied to canard explosion, J. Phys. A Math. Theor., 44: 465203.

[16] Ginoux, J.M., Llibre, J. and Chua, L.O. (2013). Canards from Chua’s circuit, Int. J. Bifurc. Chaos, 23(4): 1330010.

[17] Ginoux, J. M., \& Rossetto, B. (2014). Slow invariant manifold of heartbeat model, arXiv preprint arXiv:1408.4988.

[18] Ginoux, J. M. (2014). The slow invariant manifold of the Lorenz-Krishnamurthy model, Qualitative theory of dynamical systems, 13(1): 19-37.

[19] Anguelov, R. \& Stoltz, S. M. (2017). Stationary and oscillatory patterns in a coupled Brusselator model, Mathematics and Computers in Simulation, 133(C): 39-46.

[20] Alqahtani, AM. (2018). Numerical simulation to study the pattern formation of reaction-diffusion Brusselator model arising in triple collision and enzymatic, Journal of Mathematical Chemistry, 56(6):1543-1566.

[21] Nazimuddin, A. K. M. and Ali, M. S. (2019). Periodic Pattern Formation Analysis Numerically in a Chemical Reaction-Diffusion System, International Journal of Mathematical Sciences and Computing (IJMSC), 5(3):17-26.

[22] Nazimuddin, A. K. M. and Ali, M. S. (2019). Pattern Formation in the Brusselator Model Using Numerical Bifurcation Analysis, Punjab University Journal of Mathematics, 51(11):31-39.

[23] Prigogine, I. and Lefever, R. (1968). Symmetry breaking instabilities in dissipative systems, The Journal of Chemical Physics, 48(4), 1695-1700.

[24] Schlomiuk D. (1999). Elementary first integrals of differential equations and invariant algebraic curves , Expositiones Mathematicae, 11: 433-454. 
[25] Llibre J . \& Medrado J. C. (2007). On the invariant hyperplanes for d-dimensional polynomial vector fields , J. Phys. A. Math. Theor., 40 : 8385-8391.

[26] William E. B. \& Richard C. D. (2001). Elementary differential equations and boundary value problems, John Wiley \& Sons, Inc, New York.

\section{Authors' Profiles}

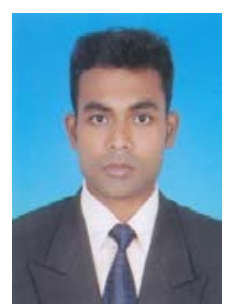

A. K. M. Nazimuddin received his B. S. (Hons) degree in Mathematics and M. S. in Applied Mathematics from the University of Dhaka. He is working as a Senior Lecturer in the Department of Mathematical and Physical Sciences, East West University, Dhaka, Bangladesh. His research interest is on Dynamical Systems, Riemannian Geometry, Symplectic Geometry and Contact Geometry.

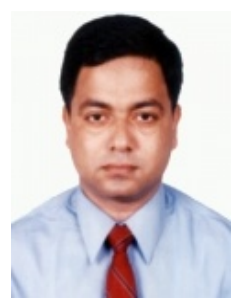

Md. Showkat Ali received his $\mathrm{PhD}$ from the University of Glasgow, Glasgow, UK. He received his B. S. (Hons) degree in Mathematics and M. S. in Applied Mathematics from the University of Dhaka, Bangladesh. He is working as a Professor in the Department of Applied Mathematics, University of Dhaka. His research interest is on Differential Geometry and Dynamical Systems. He has published about fifty research articles in this field in various reputed national and international journals.

How to cite this paper: A. K. M. Nazimuddin, Md. Showkat Ali, "Slow Invariant Manifold of Brusselator Model", International Journal of Mathematical Sciences and Computing(IJMSC), Vol.6, No.2, pp.79-87, 2020. DOI: $10.5815 /$ ijmsc.2020.02.05 